\title{
Análise do Método de Extensão Ativa da Constelação para Redução de PAPR em sistemas OFDM
}

\author{
Ian Ulian, André N. Barreto e Paulo H. P. de Carvalho
}

\begin{abstract}
Resumo-A alta razão entre a potência de pico e a potência média (PAPR) é uma das principais desvantagens de sistemas OFDM, o que diminui a eficiência de transmissão com amplificadores de potência não lineares e causa distorções indesejáveis e radiação fora de banda. Dentre os diversos esquemas propostos para se mitigar este problema merece destaque o método de extensão ativa de constelação, também chamado de método de Jones, que é bastante eficiente e não necessita nenhuma sinalização adicional. Neste artigo fazemos uma análise detalhada deste esquema, considerando seu desempenho com complexidade variável e diferentes parâmetros de otimização para diversos modelos de amplificador e diferentes esquemas de modulação.
\end{abstract}

Palavras-Chave-OFDM, PAPR, extensão ativa da constelação

Abstract-The high peak-to-average power ratio (PAPR) is one of the main disadvantages of OFDM systems, which reduces the transmission efficiency with nonlinear power amplifiers and causes unwanted distortions and out-of-band radiation. Among the many proposed schemes to mitigate this problem, the active constellation extension method, also called Jones' method, deserves some attention, as it is efficient and doesn't require any extra signalling. In this contribution we make a thorough analysis of this method, considering its performance with variable complexity and different optimisation parameters for several amplifier models and different modulation schemes.

Keywords-OFDM, PAPR, active constellation extension

\section{INTRODUÇÃO}

A modulação por múltiplas portadoras OFDM (Orthogonal Frequency Division Multiplexing) tem se tornado a base para diversos padrões de comunicação sem fio. São exemplos desse fato os padrões WiFi IEEE 802.11a [1], WiMAX IEEE 802.11e [2] e o 3GPP LTE [3], entre outros. A ideia básica da técnica OFDM é dividir os dados a serem transmitidos em diversos blocos e transmiti-los utilizando diferentes subportadoras ortogonais entre si a uma baixa taxa de transmissão. É interessante ressaltar que cada subportadora irá experimentar um canal de banda estreita, e, consequentemente, a estrutura do receptor se torna mais simples, já que a demodulação poderá ser realizada utilizando um equalizador de apenas um tap. Por este motivo sistemas OFDM são considerados robustos em relação à propagação com multipercursos, e permitem o uso de esquemas de modulação com alta eficiência espectral.

Ian Ulian e Paulo H. P. de Carvalho, Universidade de Brasília (UnB), Depto de Eng. Elétrica, Brasília-DF, E-mails:ian.ulian@hotmail.com, paulo@ene.unb.br; André N. Barreto, Instituto Nokia de Tecnologia (INdT), Brasília-DF, E-mail:andre.barreto@indt.org.br
Apesar das inúmeras vantagens, sinais OFDM apresentam uma alta variação de amplitude em sua forma de onda, usualmente representada pela razão entre a potência de pico e a média (PAPR Peak-to-Average Power Ratio) [4], o que é uma das suas principais desvantagens. Consequentemente, OFDM sofre com efeitos da não linearidade de amplificadores de potência. Para um amplificador operar em sua faixa linear com um sinal OFDM é necessário um alto valor de backoff, o que implica em pouca eficiência de potência. Por outro lado, se um baixo valor de backoff for selecionado para que haja maior eficiência de potência, o sinal será cortado devido à região de saturação do amplificador, e sofrerá com radiação fora de banda e distorções não lineares.

Existem diversas técnicas utilizadas com o intuito de diminuir a PAPR de um sinal OFDM [4], de modo que seja possível alcançar uma situação com pequenos valores de backoff e alta eficiência de potência com degradação tolerável do sinal. Grande parte desses métodos exigem porém o envio de sinalização a mais, como o método de sequências de transmissão parciais [5]-[7] e o de mapeamento seletivo [8],[9]; ou alterações no receptor para identificar o sinal OFDM original [10].

O método da extensão ativa da constelação do canal [11], também conhecido como método de Jones, consiste em um esquema para redução de PAPR simples de ser empregado e bastante eficaz. Além disso, não necessita de sinalizações adicionais, o que o torna aceitável pelas normas de tecnologias sem fio, e, apesar de alterar a constelação do sinal, não aumenta sua taxa de erro. Pelo nosso conhecimento, não existe porém na literatura uma análise detalhada da eficiência deste método, considerando, entre outros aspectos, diferentes números de iterações e diferentes modulações para diversos tipos de amplificadores. Este trabalho propõe-se a fazer este tipo de estudo. Nessas análises são levadas em consideração a taxa de erro de bit (BER) no receptor, a degradação total do sistema, o backoff utilizado e a densidade espectral de potência do sinal após o amplificador.

O artigo está estruturado da seguinte forma. Na Seção II é apresentado um modelo do sistema OFDM, dos amplificadores e a definição do PAPR. Na Seção III é descrito o método de extensão ativa de constelação. Resultados de simulação que nos permitem fazer uma análise estendida deste método são mostrados na Seção IV e na Seção V são feitas discussões finais sobre o artigo. 


\section{Modelo Do SistemA}

Em uma transmissão OFDM, os bits de entrada são divididos em blocos proporcionais ao número de subportadoras e ao tamanho da palavra designada pelo tipo de modulação de cada subportadora. Assim, um símbolo OFDM no tempo, após a execução da IFFT, consiste na soma de $N$ símbolos independentes mapeados nas $N$ subportadoras do sistema. No intervalo do $n$-ésimo símbolo OFDM ele pode ser descrito da seguinte forma

$$
s_{n}(t)=\sum_{k=1}^{N-1} x_{k, n} e^{j 2 \pi f_{k} t},
$$

em que $x_{k, n}$ é o símbolo mapeado na $k$-ésima portadora $f_{k}$.

O sinal em questão é então submetido a um amplificador de potência. Podemos representar em banda base o sinal na entrada do amplificador usando a seguinte notação

$$
s(t)=A(t) e^{j \Psi(t)},
$$

O sinal em banda base na saída do dispositivo será representado então por

$$
y(t)=G[A(t)] e^{j\{\Psi(t)+\Phi[A(t)]\}},
$$

em que $G[A(t)]$ e $\Phi[A(t)]$ representam a conversão AM/AM e $\mathrm{AM} / \mathrm{PM}$, respectivamente, causada pelo amplificador não linear.

Os modelos de amplificadores utilizados no trabalho consistem no modelo de Rapp [12] para um amplificador de estado sólido, cuja conversão AM/PM podem ser desprezada, e um limitador de envelope ideal. A conversão AM/AM do modelo de Rapp é modelada por

$$
G(A)=\frac{v A}{\left[1+\left(\frac{v A}{A_{0}}\right)^{2 p}\right]^{1 / 2 p}},
$$

sendo $A_{0}$ a amplitude máxima, $\nu$ o ganho de pequeno sinal e $p$ o fator de suavização para o modelo de Rapp. Tanto a amplitude máxima quanto o ganho de pequeno sinal foram normalizados para uma unidade.

Para um limitador de envelope ideal a conversão AM/AM é dada por

$$
G(A)=\left\{\begin{array}{ccc}
\nu A_{0} & \text { se } & A>A_{0} \\
\nu A & \text { se } & A \leq A_{0}
\end{array} .\right.
$$

A Figura 1 evidencia a função de transferência de amplitude dos amplificadores em questão, para os modelos de Rapp foram utilizados os valores de $p=2$ e $p=3$.

Nota-se que quanto maior é o valor de $p$ mais suave é a transição para a saturação.

Além disso, em uma análise envolvendo amplificadores, a definição de backoff de entrada (IBO) e de saída (OBO) se torna indispensável:

$$
\begin{gathered}
I B O_{d B}=10 \log _{10}\left(\frac{P_{\text {sat }, \text { in }}}{P_{\text {in }}}\right) \\
O B O_{d B}=10 \log _{10}\left(\frac{P_{\text {sat }, \text { out }}}{P_{\text {out }}}\right),
\end{gathered}
$$

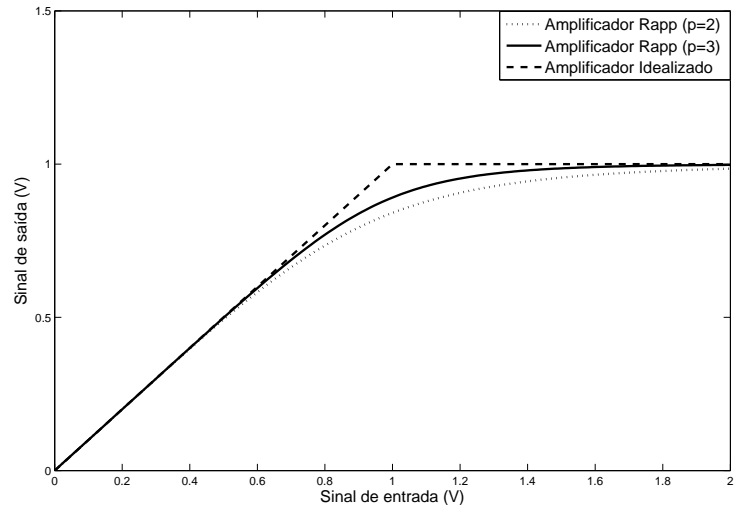

Fig. 1. Função de Transferência dos Amplificadores

em que $P_{\text {in }}$ e $P_{\text {out }}$ são as potências médias de entrada e saída, respectivamente; e $P_{\text {sat, in }}$ e $P_{\text {sat, out }}$ são as potências de saturação de entrada e saída, respectivamente, que são obtidas da função de conversão AM/AM.

É importante salientar que amplificadores de potência são mais eficientes quando trabalham perto da potência de saturação. Analisando novamente a Figura 1, nota-se que se o sinal possuir uma amplitude constante, é possível trabalhar perto da saturação com alta eficiência de potência.

Entretanto, como vemos em (1), um sinal OFDM corresponde à soma de diversas portadoras, que, dependendo dos símbolos transmitidos, se somam ou se cancelam. Desta forma um sinal OFDM não possui amplitude constante, muito pelo contrário, nele observa-se uma grande variação na amplitude. Ou seja, sinais OFDM apresentam altos valores de razão entre a potência de pico e a potência média (PAPR). Desta forma, ou escolhe-se um nível de operação bem abaixo da potência de saturação, para que mesmo os picos mais altos sejam amplificados linearmente, porém com baixa eficiência média; ou trabalha-se perto do nível de saturação e aceita-se que parte do sinal com amplitude mais alta sofra seja distorcida. Além disso, é sabido que distorções não lineares causam radiação fora de banda.

A PAPR de um sinal OFDM analógico em um símbolo OFDM de duração $T_{s}$ é definida como:

$$
\operatorname{PAPR}(s(t))=\frac{\max _{0 \leq t \leq T_{s}}|s(t)|^{2}}{E\left[\frac{1}{T_{s}} \int_{0}^{T_{s}}|s(t)|^{2} d t\right]}
$$

e é uma métrica comumente utilizada para a variabilidade de amplitude de um sinal. Portanto, para trabalharmos eficientemente com baixa distorção com amplificadores não lineares é desejável reduzirmos ao máximo o PAPR, mantendo as características positivas de um sinal OFDM.

\section{O MÉTODO DE EXTENSÃO ATIVA DA CONSTELAÇÃO}

O método de extensão ativa da constelação[11] consiste na modificação da constelação do sinal sem aumentar sua taxa de erro. $\mathrm{O}$ efeito dessa modificação é a adição de senóides ou cossenóides em algumas frequências que podem acarretar no cancelamento de alguns picos do sinal. A alteração da 
constelação não compromete o desempenho, uma vez que os pontos sinais modificados são projetados em uma região com maior margem de erro.

A Figura 2 mostra a constelação de um sinal com a modulação 16 QAM ao ser modificado pelo método em questão, na qual é possível perceber que as regiões ótimas de decisão não são abandonadas.

Além disso, é interessante notar que a aplicação do método acarreta entretanto no aumento da potência do sinal. Porém, esse aumento não é significativo quando comparado ao ganho trazido por ele [11].

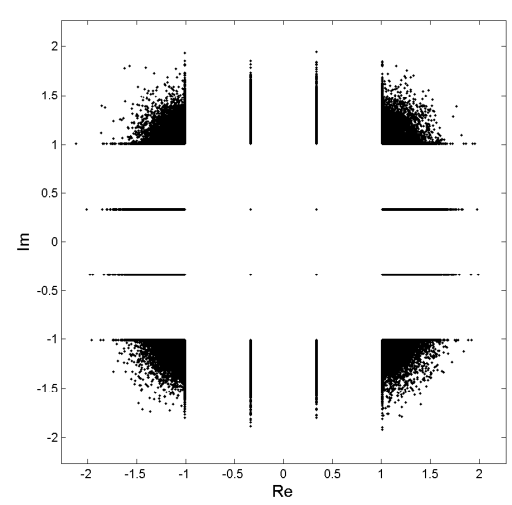

Fig. 2. Constelação após aplicação do método de Jones para o 16 QAM

O algoritmo deve modificar apenas as subportadoras de dados, deixando inalteradas as subportadoras piloto e de guarda. Ele pode ser descrito pelos seguintes passos, que são executados símbolo a símbolo OFDM:

1) De acordo com os dados de entrada, designar os pontos $x_{k}$ da constelação

2) Construir o símbolo no tempo via IFFT

3) Comparar a magnitude de todas as amostras com $L_{\max }$, o valor estipulado como máximo

4) Para as amostras que excederam $L_{\max }$, redimensionálas, isto é, fazer $\tilde{x}=L_{\max } e^{j \theta_{n}}$, sendo $x_{n}=\left|x_{n}\right| e^{j \theta_{n}}$

5) Obter novamente o sinal no domínio da freqüência via FFT

6) Restabelecer as portadoras piloto e de guarda. Restaurar os pontos interiores aos valores originais e projetar os pontos externos para as regiões de maior margem

7) Retornar ao passo 2 e executar o algoritmo até que nenhuma amostra temporal seja redimensionada ou que o número máximo de iterações seja atingido

Além das vantagens já citadas do método, percebe-se que seu algoritmo não possui grande complexidade e, como será visto, não são necessárias muitas iterações para que se chegue a um resultado satisfatório. Outra característica extremamente positiva consiste no fato de que sua aplicação não demanda sinalização adicional, ou nenhuma modificação nos receptores. Isso o torna aceitável pelas normas que definem tecnologias que empregam a transmissão OFDM em sua camada física.

É interessante ressaltar, também, que os únicos parâmetros ajustáveis do método são o número de iterações máximo e o valor de $L_{\max }$, as quais serão analisadas na próxima Seção.

\section{Resultados}

Nesta Seção será analisado o desempenho do método da modificação ativa da constelação em diferentes situações. Foi simulado um sistema OFDM com 512 subportadoras, das quais 420 transmitem dados. A subportadora central (DC) e as mais externas são mantidas nulas para conformação do espectro. $\mathrm{O}$ espaçamento entre as subportadoras é de $15 \mathrm{kHz}$ e o prefixo cíclico corresponde a 1/16 do tamanho útil do símbolo. Todos os resultados abaixo foram obtidos considerando um sinal amostrado a uma taxa 4 vezes maior que a amostragem crítica do sinal OFDM.

A Figura 3 mostra três curvas de CDF de PAPR em um sinal OFDM, na qual é possível observar que a utilização do método realmente diminui a probabilidade de se encontrar altos valores de pico no sinal. Também podemos ver que a diminuição do PAPR é maior quanto menor for o valor $L_{\max }$ selecionado para limitarmos a amplitude do sinal.

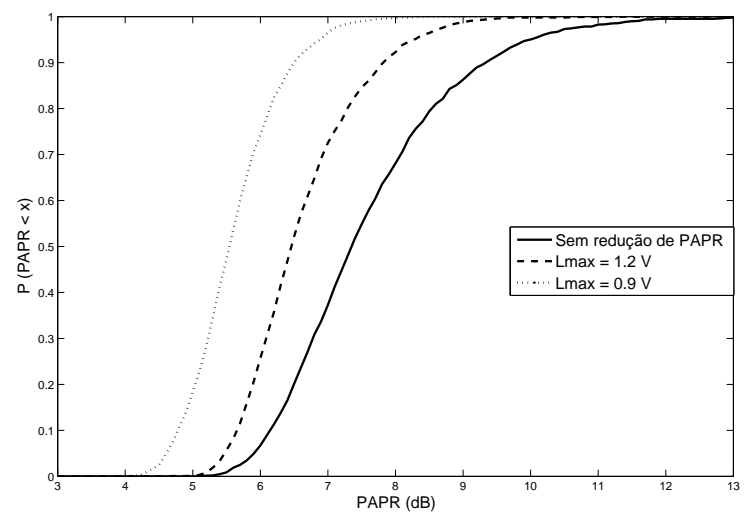

Fig. 3. CDF de PAPR de um sinal OFDM sem e com redução de PAPR variando o valor de Lmax

Entretanto, não é suficiente apenas reduzir a PAPR de um sinal, deve-se garantir, também, o bom desempenho do sistema. Potanto, a análise do desempenho de um sistema OFDM quando se leva em consideração a presença de um amplificador não linear deve ser feita à sombra de três quesitos: a taxa de erro de bit (BER), o backoff utilizado e a radiação fora de banda causada.

Entretanto, não tem sentido analisar o backoff isoladamente. Uma avaliação mais inteligente consiste na determinação da degradação total (DT) de um sistema, que é definida por

$$
D T_{d B}=O B O_{d B}+\left[E_{b} / N 0_{(O B O)}-E_{b} / N_{0_{(\text {linear })}}\right]_{B E R}
$$

em que $E_{b} / N_{0}(O B O)$ e $E_{b} / N_{0}$ (linear) são as razões de energia de bit $E_{b}$ por densidade espectral de potência do ruído $N_{0}$ requeridas, para um determinado $\mathrm{BER}$, em $\mathrm{dB}$ para o amplificador não linear utilizado e para um amplificador ideal linear, e $O B O_{d B}$ é o backoff de saída. Ou seja, o primeiro termo nos indica a degradação causada pela diminuição da potência de transmissão potencial do amplificador, enquanto que o segundo termo representa a degradação devido à distorção não linear causada pelo amplificador. Além disso, deve-se ressaltar que essa figura de mérito é mensurada para um determinado canal 
e para uma determinada BER. Em todas as análises que serão feitas a seguir são considerados um canal AWGN e uma BER de $10^{-4}$. Um exemplo de curva de degradação total pode ser visto na Figura 8

A análise de uma curva de degradação total permite chegar a um valor de backoff ótimo, que é o valor para o qual se tem a menor degradação do sistema.

Além desse parâmetro de análise, será levada em conta a ACPR (Adjacent Channel Power Ratio), que consiste numa relação entre quanto da potência do sinal está dentro da banda $\left(P_{i n B a n d}\right)$ e quanto da potência está fora da banda original $\left(P_{\text {outBand }}\right)$. A ACPR é definida a seguir e será analisada em função do backoff de saída.

$$
A C P R_{d B}=10 \log _{10}\left(\frac{P_{\text {inBand }}}{P_{\text {outBand }}}\right) \text {, }
$$

Primeiramente, a atuação do método será verificada para diferentes números de iterações considerando diferentes tipos de modulações. Para isso, foi gerada uma curva de degradação total para diferentes números de iterações. Para cada curva gerada, obteve-se um valor de backoff ótimo, ou seja, obtevese um backoff ótimo para cada número máximo de iterações. Esse resultado é mostrado na Figura 4, que foi obtida em uma simulação com o amplificador modelo Rapp com $p=3$ e com $L_{\max }=1 \mathrm{~V}$ para o método de Jones.

Ao analisá-la é possível verificar que quanto maior o número de iterações utilizado, melhor o resultado obtido levando em consideração o OBO ótimo. No entanto, não é de grande valia utilizar um número máximo de iterações muito grande, uma vez que o ganho obtido com ele não é alto em relação a um menor número de iterações. Deve-se portanto escolher um número menor, pois o gasto computacional com o método é inferior. Para simulações posteriores será considerado o número ótimo de iterações aquele que garantir cerca de $0.1 \mathrm{~dB}$ abaixo do ganho com o maior número de iterações analisados.

Analisando a curva obtida para a modulação QPSK, notase que com 20 iterações obteve-se um ganho de $0.87 \mathrm{~dB}$. No entanto, ao se utilizar 10 iterações, obteve-se um ganho de 0.76 dB. Portanto, deve-se optar por utilizar 10 iterações, visto que possui menor esforço computacional do que 20 e considerando que os ganhos são bem próximos.

Já para o 16 QAM, com 20 iterações se observa um ganho de $0.88 \mathrm{~dB}$ e com 11 , um ganho de $0.83 \mathrm{~dB}$. Logo, deve-se optar por 11 iterações pelo mesmo motivo que se optou por 10 para a modulação QPSK.

Para a modulação 64 QAM, percebe-se um ganho de 0.16 $\mathrm{dB}$ com 20 iterações, e de $0.09 \mathrm{~dB}$ para 7 iterações. Nota-se que a aplicação do método para esse caso não é muito vantajosa, pois o ganho obtido é muito pequeno ao se considerar o aumento do esforço computacional. Esse ganho baixo consiste no fato de que para essa modulação a minoria dos pontos da constelação podem ter suas posições alteradas.

A Figura 5 evidencia a degradação total mínima obtida em função do número máximo de iterações. Pode ser observado um comportamento semelhante às curvas de backoff mínimo, ou seja, são necessárias apenas poucas iterações para o método convergir.

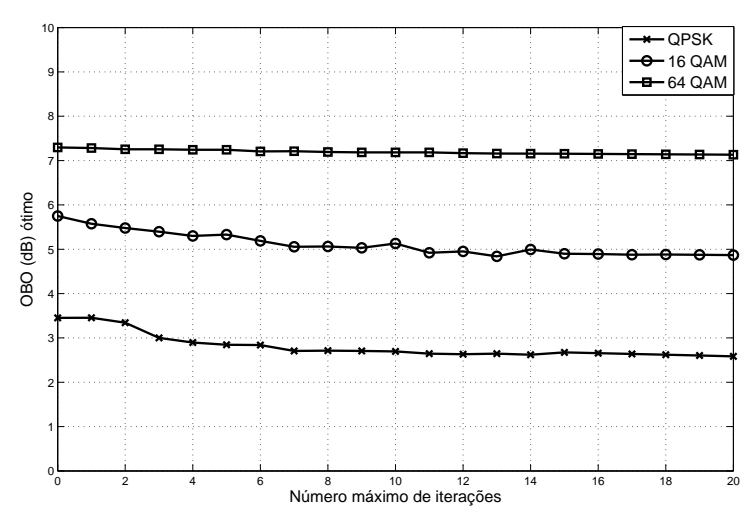

Fig. 4. OBO ótimo em função do número máximo de iterações

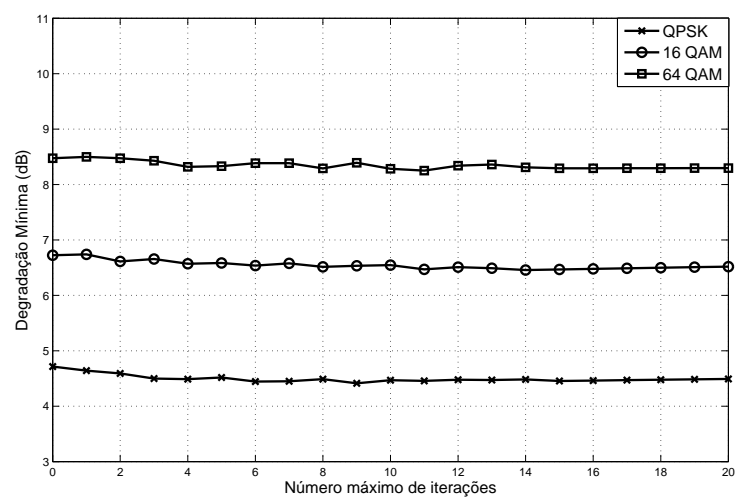

Fig. 5. Degradação total mínima em função do número máximo de iterações

O outro parâmetro ajustável do método de Jones é o valor de $L_{\max }$. Foram portanto realizadas simulações com a finalidade de se encontrar um valor ideal de $L_{\max }$ para cada amplificador, já que eles possuem comportamentos diferentes. O valor considerado ideal será aquele que produzir o menor valor para o OBO ótimo. As simulações foram realizadas para os tipos de amplificadores com respostas evidenciadas na Figura 1. Os resultados de simulação se encontram na Figura 6, que foi gerada para uma simulação com modulação 16 QAM e 10 iterações do método de Jones.

Ao analisá-la para o modelo de Rapp com $p=2$, obteve-se para o melhor caso um OBO $=5.82 \mathrm{~dB}$ para $L_{\max }=0.8 \mathrm{~V}$. Utilizando $p=3$ para o mesmo modelo, chegou-se a um valor de $\mathrm{OBO}=5.02 \mathrm{~dB}$ com $L_{\max }=0.9 \mathrm{~V}$ para o melhor caso. Já para o amplificador idealizado, com um valor de $L_{\max }=$ $1 \mathrm{~V}$, obteve-se $\mathrm{OBO}=4.22 \mathrm{~dB}$.

Portanto, nota-se que quanto mais próximo do ideal é o comportamento do amplificador, menor é o backoff ótimo e maior o valor de $L_{\max }$ a ser utilizado na aplicação do método, at;e um máximo de $L_{\max }=1$. É também interessante ressaltar que para um amplificador idealizado existe claramente um menor backoff ótimo para exatamente $L_{\max }=1$, o que já poderia se esperar pelo comportamento do amplificador, que nada mais que é um limitador neste nível.

A Figura 7 ilustra a degradação total mínima obtida em 
função do valor de $L_{\max }$. Neste caso vimos que, mesmo para o amplificador idealizado, o ótimo é limitar o sinal a um valor $L_{\max }<1$.

É importante ressaltar aqui que o algoritmo de redução de PAPR é aplicado no sinal amostrado à taxa de saída da IFFT, e que ocorre crescimento dos picos após a filtragem do sinal. Desta forma, não é garantido que o sinal super-amostrado ainda estará limitado ao valor $L_{\max }$ selecionado.

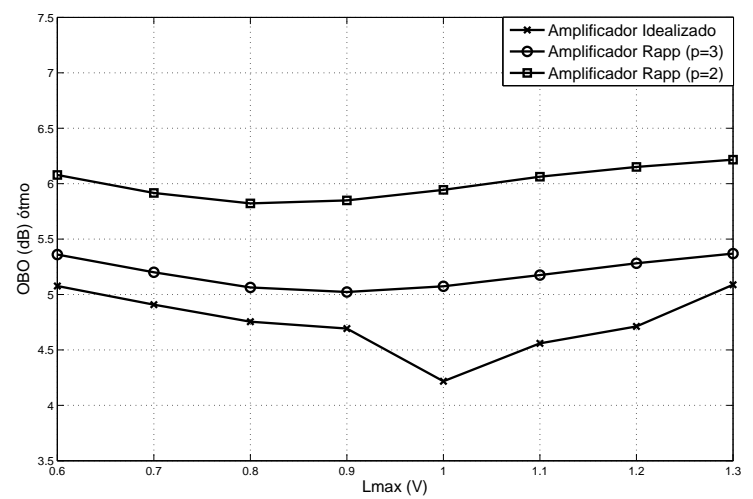

Fig. 6. OBO ótimo em função do valor de $L_{\max }$

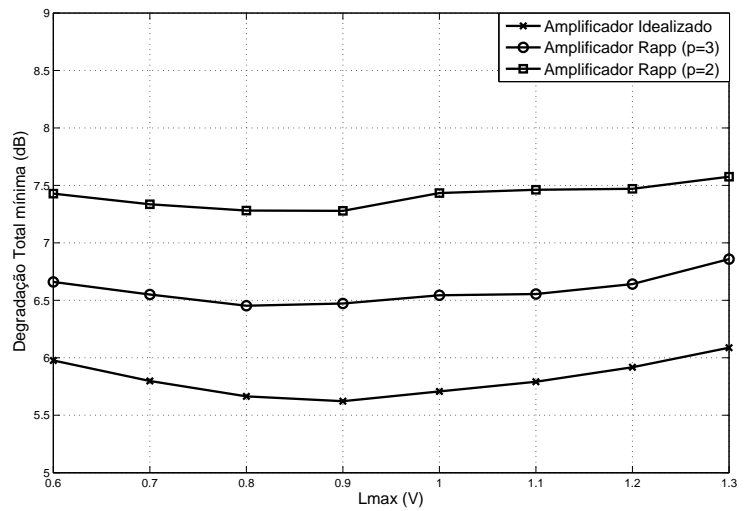

Fig. 7. Degradação total mínima em função do valor de $L_{\max }$

Considerando o amplificador modelo de Rapp com $p=$ 3 , tem-se um valor ideal para $L_{\max }$ e ,para as diferentes modulações, tem-se um numero ótimo para o numero máximo de iterações. Logo, com essas informações deseja-se chegar ao maior ganho que o método pode proporcionar para o amplificador em questão e para as diferentes modulações analisadas no trabalho.

Na Figura 8 é possível verificar em termos de degradação total a utilização do método de redução de PAPR detalhado aqui. Ela ilustra a situação em que não há redução de PAPR e a situação na qual o método é aplicado para as três modulações consideradas aqui. É interessante ressaltar que para o número de iterações foi utilizado o valor ótimo encontrado ao se analisar a Figura 4. Além disso, $L_{\max }=0.9 \mathrm{~V}$, visto que foi utilizado o modelo de Rapp com $p=3$ para o amplificador.

Analisando o caso da modulação QPSK, observa-se um ganho de $0.64 \mathrm{~dB}$ em relação ao $\mathrm{OBO}$ e um ganho de 0.36 $\mathrm{dB}$ para a degradação total. Além disso, existe um acréscimo de $0.23 \mathrm{~dB}$ de ACPR.

Para o 16 QAM, tem-se um ganho de $1.09 \mathrm{~dB}$ de OBO e de $0.34 \mathrm{~dB}$ de degradação. Considerando a ACPR, nota-se uma diminuição de $1.43 \mathrm{~dB}$.

Utilizando 64 QAM, chega-se a $0.48 \mathrm{~dB}$ de ganho para o OBO e a $0.18 \mathrm{~dB}$ para a degradação total. Nota-se, também, que há um acréscimo de $0.35 \mathrm{~dB}$ para ACPR.

Logo, o método utilizado com os parâmetros otimizados continua trazendo ganho e somente para o caso em que se utilizou 16 QAM a ACPR diminui. Porém, foi o caso em que se obteve maior ganho considerando o OBO.

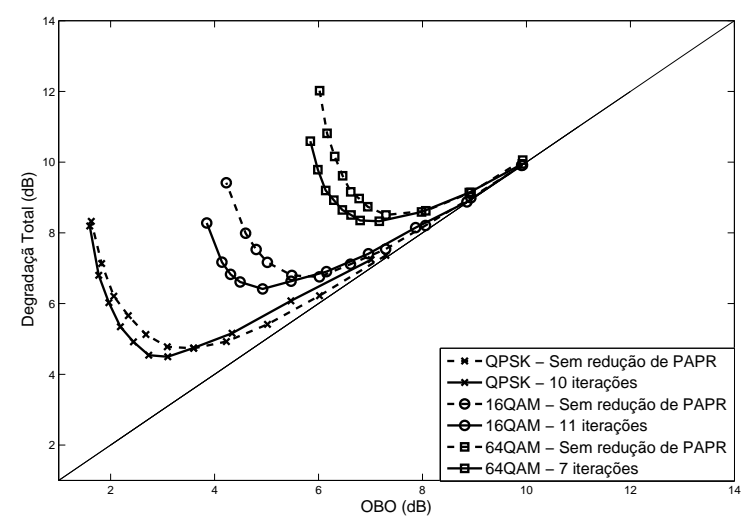

Fig. 8. Degradação total utilizando QPSK, 16QAM e 64QAM

A Figura 9 mostra o espectro de sinais OFDM modulados em 16 QAM e que utilizam 512 subportadoras. Nela é possível visualizar um sinal sem distorção não linear e sinais com e sem redução de PAPR com valores de backoff ótimo regidos pela Figura 8. Além disso, a distorção não linear é causada por um amplificador que segue o modelo de Rapp com $p=3$.

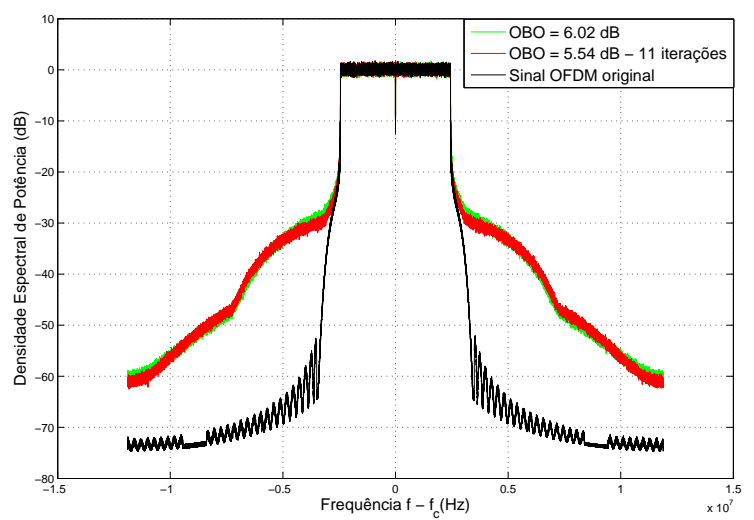

Fig. 9. Espectro de um sinal OFDM 16 QAM com e sem redução de PAPR, Amplificador Rapp $p=3$

\section{CONCLUSÕES}

Neste artigo fizemos uma análise estendida do esquema de redução de PAPR por meio da extensão ativa da constelação, 


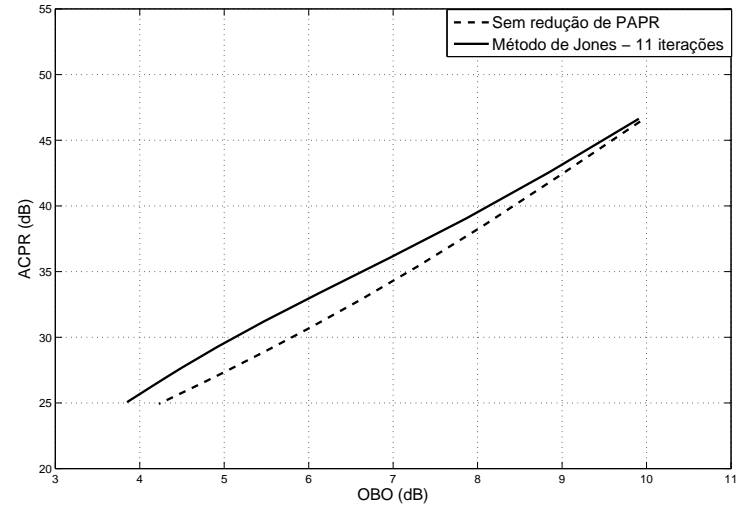

Fig. 10. Variação do ACPR para diferentes valores de OBO

e vimos que este método é bastante eficiente mesmo com poucas iterações. Entretanto, seu desempenho não é tão bom quando esquemas de modulação de ordem mais elevada são considerados. Também fizemos uma análise do impacto do limite de amplitude $L_{\max }$ no desempenho e vimos que uma escolha adequada deste parâmetro pode também trazer benefícios, e que esta escolha pode depender do modelo de amplificador utilizado. Por não necessitar de modificações nas normas vigentes, este método se mostra bastante promissor em sistemas padronizados como o WiMAX e o LTE. Por esse motivo, nossos trabalhos futuros se concentrarão no estudo da redução do PAPR nestes sistemas.

\section{AgRAdecimentos}

Este trabalho foi parcialmente financiado pelo CNPq através do projeto WiPA, Processo 554047/2006-3.

\section{REFERENCES}

[1] IEEE Std 802.11a-1999, " Part 11: Wireless LAN Medium Access Control (MAC) and Physical Layer (PHY) specifications - High-speed Physical Layer in the $5 \mathrm{GHz}$ Band"

[2] IEEE Standard 802.16e-2005, "IEEE Standard for Local and metropolitan area networks Part 16: Air Interface for Fixed Broadband Wireless Access Systems,'May 2004.

[3] H. Ekstrom et al., "Technical solutions for the 3G Long Term Evolution", IEEE Commun. Mag., pp. 38-44, Mar. 2006.

[4] H. Ochiai and H. Imai, "Performance analysis of deliberately clipped OFDM signals," IEEE Trans. Commun., vol. 50, pp. 89-101, Jan. 2002

[5] S. H. Mueller e J.B. Huber, "OFDM with reduced peak-to-average power ratio by optimum combination of partial transmit sequences,'Electronics Letters, vol. 33, no. 5, pp. 368-369, Feb. 1997.

[6] L. J. Cimini, Jr. e N. R. Sollenberger, "Peak-to-average power ratio reduction of an OFDM signal using partial transmit sequences with embedded side information," in Proc. Globecom 2000

[7] T. Jiang, W. Xiang, P.C. Richardson, J. Guo e G. Zhu, "PAPR Reduction of OFDM Signals Using Partial Transmit Sequences With Low Computational Complexity," in IEEE Trans. Broadcasting, 53(3), set. 2007

[8] A. D. S. Jayalath e C. Tellambura, ”Reducing the peak-to-average power ratio of orthogonal frequency division multiplexing signal through bit or symbol interleaving," in Electronics Letters, vol. 36, no. 13, pp. 1161-1163, junho 2000.

[9] Z. Du, N. C. Beaulieu e J. Zhu, "Selective Time-Domain Filtering for Reduced-Complexity PAPR Reduction in OFDM," in IEEE Trans. Veh. Tech., 58(3), mar. 2009

[10] X. Wang, T. T. Tjhung e C. S. Ng, "Reduction of Peak-to-Average Power Ratio of OFDM System Using a Companding Technique," in IEEE Trans. Broadcasting, 45(3), set. 1999
[11] B. S. Krongold and D. L. Jones, "PAR Reduction in OFDM via Active Constellation Extension," in IEEE Trans. broadcasting, vol 49, no 3, set. 2003, pp.25

[12] C. Rapp, "Effects of HPA-Nonlinearity on a 4-DPSK/OFDM-Signal for a Digitial Sound Broadcasting System," in Proc. 2ndd European Conf. Satellite Commun., Liege, Belgium, out. 22-24, 1991, pp. 179-184. 